\title{
Cinética Enzimática del Bagazo de Caña para la Producción de Glucosa Utilizando la Enzima Trichoderma Iongibrachiatum
}

Diofanor Acevedo ${ }^{(1) \star}$, Clemente Granados ${ }^{(1)}$ y Eliana M. Guerrero(2)

(1) Universidad de Cartagena, Facultad de Ingeniería, Departamento de Ingeniería de Alimentos, Avenida el Consulado, Calle 30 No. 48-152. Cartagena, Bolívar-Colombia (e-mail: diofanor3000@gmail.com).

(2) Universidad de San Buenaventura, Facultad de Ingeniería Química, Calle Real de Ternera No. 30-966. Cartagena, Bolívar-Colombia.

${ }^{*}$ Autor a quien debe ser dirigida la correspondencia

Recibido Ene. 17, 2014; Aceptado Abr. 19, 2014; Versión final recibida May. 21, 2014

\section{Resumen}

Se ha estudiado la hidrólisis enzimática del bagazo de caña utilizando la enzima Trichoderma longibrachiatum. Siguiendo la cinética de velocidad propuesta por Michaelis-Menten, de los datos de $V_{0}$ y $S_{0}$ obtenidos a concentraciones de 30,40 y $50 \mathrm{mg} / \mathrm{mL}$ de bagazo de caña con dosificaciones de enzima constante de $0.210,0.420$ y $0.840 \mathrm{mg} / \mathrm{mL}$ se calcularon los valores de $\mathrm{K}_{\mathrm{m}}$ y $\mathrm{V}_{\max }$. A concentración de sustrato de $50 \mathrm{mg} / \mathrm{mL}$ y de enzima de $0,840 \mathrm{~mL}$ se obtuvo la mayor concentración de glucosa $(0.1902$ $\mathrm{mg} / \mathrm{mL}$ ). El valor de $K_{\mathrm{m}}$ para la enzima fue de $22.8 \mathrm{mg} / \mathrm{mL}$ lo que indica que tiene poca afinidad con el sustrato. Los valores de $\mathrm{V}_{\max }$ para las diferentes dosificaciones de enzima fueron de 0.08495, $0.1034 \mathrm{y}$ $0.1176 \mathrm{mg} / \mathrm{mL} \cdot \mathrm{s}$.

Palabras clave: cinética enzimática, bagazo de caña, Trichoderma longibrachiatum, Michaelis-Menten

\section{Enzyme Kinetics of Sugarcane Bagasse for Glucose Production using Enzyme Trichoderma longibrachiatum}

\begin{abstract}
The enzymatic hydrolysis of sugarcane bagasse using enzyme Trichoderma longibrachiatum has been studied. Following the kinetic rate proposed by Michaelis-Menten, data of $V_{0}$ and $S_{0}$ obtained at concentrations of 30,40 and $50 \mathrm{mg} / \mathrm{mL}$ of bagasse with constant dosages of enzyme of $0.210,0.420$ and $0.840 \mathrm{mg} / \mathrm{mL}$, values of $\mathrm{Km}$ and $V_{\max }$ were calculated. With substrate concentration of $50 \mathrm{mg} / \mathrm{mL}$ and 0.840 $\mathrm{mL}$ of enzyme, the greater glucose concentration was obtained $(0.1902 \mathrm{mg} / \mathrm{mL})$. The value of $\mathrm{K}_{\mathrm{m}}$ for the enzyme was $22.8 \mathrm{mg} / \mathrm{mL}$, indicating that it has little affinity for the substrate. $V_{\max }$ values for different enzyme dosages were $0.08495,0.1034$ and $0.1176 \mathrm{mg} / \mathrm{mL} \cdot \mathrm{s}$.
\end{abstract}

Keywords: enzyme kinetics, bagasse, Trichoderma longibrachiatum, Michaelis-Menten model 


\section{INTRODUCCIÓN}

El género Trichoderma son hongos saprofitos del suelo y la madera, de crecimiento muy rápido. Las especies de este género se encuentran ampliamente distribuidas por todas las latitudes, y se presentan naturalmente en diferentes ambientes, especialmente en aquellos que contienen materia orgánica 0 desechos vegetales en descomposición. En los últimos años se han incrementado los estudios de campo para su uso en cultivos de hortalizas y ornamentales. No obstante, la información sobre su empleo en la producción agrícola es insuficiente y dispersa. La capacidad de producir diversos metabolitos y de adaptación a diversas condiciones ambientales y sustratos, confiere a Trichoderma la posibilidad de ser utilizado en la industria biotecnológica. Los productos a partir de cepas seleccionadas de Trichoderma pueden ser aplicados bajo diferentes condiciones (Martínez et al., 2013). Estudios anteriores han determinado el enorme potencial de los aislados de hongos $T$. longibrachiatum en la degradación de los HAP de alto peso molecular tales como BAA y demás estudios de la industria biotecnológica (Rosales et al., 2013).

En los últimos años, ha habido una tendencia creciente hacia la utilización más eficiente de los residuos agroindustriales, incluyendo el bagazo de la caña de azúcar. Varios procesos y productos han sido reportado que utilizan bagazo de caña como materia prima. Estos incluyen la producción de la disolución de pulpa, pasta de papel, el etanol y energía, además es un material lignocelulósico con potencial para la disolución de la producción de celulosa, especialmente cuando se integra en los procesos de biorrefinería (Wolf, 2011). El bagazo es la fibra residuo restante cuando se presiona la caña de azúcar para extraer el azúcar. El bagazo se compone de fibra y médula, la fibra es de paredes gruesas y relativamente largo $(1,4$ $\mathrm{mm}$ ) (Aigbodion et al., 2010). El bagazo es un residuo lignocelulósico abundante se encuentran típicamente en los países tropicales que procesan la caña de azúcar, tales como Brasil, India, Cuba, China y Nigeria (Aigbodion et al., 2010). Las fibras de bagazo (BF) son generalmente gruesas y rígidas. Se utiliza como combustible para calderas por la fábrica de azúcar o como una materia prima para la fabricación de productos de pasta y de papel (Agunsoye y Aigbodion, 2013). Consta de aproximadamente el $40-50 \%$ de celulosa y $20-30 \%$ de hemicelulosa y $18-25 \%$ de lignina. Debido a su bajo contenido en cenizas (1-3\%), el bagazo ofrece numerosas ventajas en comparación con otros residuos de los cultivos, tales como paja de arroz, paja de trigo (Freitas y Colodette, 2014). El bagazo de caña de azúcar es un material lignocelulósico con potencial para la disolución de la producción de celulosa, especialmente cuando se integra en los procesos de biorrefinería (Wolf, 2011).

Caspeta et al., (2014) estudiaron la hidrólisis enzimática con cargas de alto contenido en sólidos para la conver-sión de bagazo de agave a etanol. Estos reportaron un método eficaz que permite la producción de jarabes con concentraciones de glucosa altos, así como los altos rendimientos de biomasa en azúcaresconversión de los residuos de bagazo de agave utilizando hidrólisis enzimática con cargas de alto contenido en sólidos realizados en un biorreactor de peg-mezclador a escala de laboratorio. Y por otro lado Sposina et al., (2013) estudiaron el uso de celulasa-celobiohidrolasa libre de mezcla para la hidrólisis de la celulosa microcristalina y el bagazo de caña de azúcar tratada previamente por moliendal, encontrando que los tratamientos previos que alteran la estructura nativa y/o la composición y/o cristalinidad de la pared celular vegetal aumentan la hidrólisis enzimática. En general, 1 tonelada métrica de caña de azúcar genera $280 \mathrm{~kg}$ de bagazo de caña, aproximadamente el $50 \%$ de este residuo se utiliza en las fábricas de azúcar/destilerías como la fuente de energía mientras que el restante se convierte en abono o utilizan en la industria de papel (Peng et al., 2009).

El principal problema en el uso de celulosa de bagazo es su estructura cristalina que hace que sea difícil para hidrolizar (Panagiotopoulos et al., 2013).Para la hidrólisis enzimática del material lignocelulósico, la mayoría de los estudios utilizaron las enzimas comerciales caros que se suman al coste de la fermentación y por lo tanto limitan la comercialización de la producción de hidrógeno a base de celulosa (Stork et al., 2009). La hidrólisis enzimática y la fermentación se pueden llevar a cabo por diversos enfoques pero con ciertos compromisos y desventajas. Hay varios estudios relacionados con la cinética enzimática de caña de azúcar (Lo et al., 2008, Pattra et al., 2008, Lo et al., 2009 y Fangkum y Reungsang, 2011), pero esta no se ha estudiado utilizando la enzima $T$. Longibrachiatum, como se presenta en este trabajo.

\section{MATERIALES Y MÉTODOS}

Materia prima: Cuatro mil cien gramos (4100 gramos) de bagazo de caña proveniente del municipio de Sahagún (Córdoba) fueron triturados con el fin de disminuir su tamaño y así hacer el material mas accesible a la hidrólisis (Sposina et al., 2013). Siguiendo la metodología de Pattra et al., (2008) se almacenó en un medio refrigerado y posteriormente se tamizó obteniendo tamaños de partícula menores de $2,4 \mathrm{~mm}$. Se secó el bagazo en un horno a $80 \stackrel{\circ}{\mathrm{C}}$ durante 24 horas. 
Hidrólisis enzimática: La enzima que utilizada corresponde a un preparado comercial de celulasa ácida suministrado por DYADIC INTERNATIONAL, INC. Con la denominación comercial ROCKSOFTTM SUPER ACE, producto \# 120. Es un preparado de celulasa líquida proveniente de una cepa de T. longibrachiatum. La enzima fue utilizada en su forma comercial. Los tratamientos enzimáticos sobre el bagazo se realizaron en condiciones óptimas de trabajo para la enzima, correspondiente a la temperatura de $40{ }^{\circ} \mathrm{C}$ y $\mathrm{pH}$ de 6 (Controlado por pH Indikator papier Universalin dikator pH 1/10 marca Merck), manteniendo agitación constante durante la hidrólisis, para lo que se utilizaron recipientes de $1000 \mathrm{ml}$ (Schott Duran), donde se adicionaron 15, 20 y $25 \mathrm{~g}$ de bagazo en $500 \mathrm{ml}$ de agua destilada. Este recipiente fue sumergido en un baño termostático a $40 \stackrel{\circ}{\circ}$ para mantener la temperatura constante durante todo el proceso. Cuando se alcanzó la temperatura dentro del recipiente se agregó el complejo enzimático con dosificaciones de 0,210,0,420 y $0,840 \mathrm{~mL}$ a concentraciones de bagazo de 30,40 y $50 \mathrm{mg} / \mathrm{mL}$ con agitación constante durante 5 horas. Las experiencias se efectuaron por duplicado, siendo el resultado final la media de los resultados obtenidos. El seguimiento de la velocidad de la hidrólisis enzimática fue controlado por medio de la determinación del contenido de glucosa soluble producido en el baño residual a diferentes tiempos. Para ello se utilizo GLUCOSA OXIDASA/PEROXIDASA (enzimática-espectrofotométrica), como reactivo sensible. Se empleó glucosa anhidra como reactivo patrón para la obtención de la curva de calibrado del método. Se utilizó un espectrofotómetro Spectronic 20D+ para la lectura de la absorbancia.

Preparación de la curva de calibrado y determinación del contenido de glucosa: La curva de calibrado se determinó tomando como referencia el reactivo anhidro de glucosa. Se prepararon disoluciones con concentraciones conocidas de 0,4 a $0,004 \mathrm{mg}$ de glucosa $/ \mathrm{mL}$, determinándose la absorbancia según el procedimiento analítico experimental descrito por Guerrero (2004). Para determinar el contenido de glucosa de las muestras extraídas durante la hidrólisis enzimática, se procedió de la misma forma que para la preparación de la curva de calibrado, aplicando el método de análisis sobre la muestra del baño cada hora durante 5 horas. Las muestras fueron sometidas a filtrados para que no existieran partículas suspendidas con el objetivo de mejorar la lectura en el espectrofotómetro.

Velocidad inicial de reacción $\left(V_{0}\right)$, constante de Michaelis-Menten $\left(K_{m}\right)$ y velocidad máxima de reacción $V_{\text {max: }}$ : Habiendo obtenido las curvas de concentración de glucosa en función del tiempo se siguió con la obtención de los datos de $V_{0}$ para cada una de las concentraciones de sustrato inicial $\left(\mathrm{S}_{0}\right)$ conocidas. Para esto se debe tener en cuenta que la medida de la $V_{0}$ se debe hacer cuando la reacción enzimática esta en el estado de saturación, en donde los centros activos de la enzima se encuentran llenos de sustrato para evitar el error introducido por el deterioro de la enzima, por lo tanto la $V_{0}$ se realizo antes que se consumiera el $10 \%$ de sustrato que es la pendiente de la curva de avance a un tiempo menos de 30 minutos. Como la dosificación de enzima es muy pequeña y la $\mathrm{S}_{0}$ es muy grande al comienzo de la reacción se considera que la concentración de enzima-sustrato es constante para toda la reacción. Siguiendo la cinética de velocidad propuesta por Michaelis-Menten, de los datos de $V_{0}$ y $S_{0}$ obtenidos a concentraciones de 30,40 y $50 \mathrm{mg} / \mathrm{mL}$ de bagazo de caña con dosificaciones de enzima constante de $0,210,0,420$ y $0,840 \mathrm{mg} / \mathrm{mL}$ se calcularon los valores de $\mathrm{K}_{\mathrm{m}}$ y $\mathrm{V}_{\max }$. Para las representaciones gráficas Michaelis-Menten se utilizó el programa Hiperbolic Regresión Setup.

\section{ANÁLISIS DE RESULTADOS}

\section{Curva de calibrado y de avance de la reacción}

En la Figura 1 se representa gráficamente la curva de calibrado para los valores medios de la absorbancia. Come se puede observar, la curva de calibrado tiene un comportamiento lineal dentro de los márgenes establecidos experimentalmente. A partir de la Ecuación (1), proveniente del despeje de la concentración de glucosa de la ecuación lineal facilitada por la curva de calibrado se obtuvieron los datos de concentración de glucosa en el proceso de hidrólisis. En la Figura 2 se muestran las curvas de avance de la reacción que representa la velocidad de formación del producto en función del tiempo con dosificación de enzimas de $0,210,0,420$ y $0,840 \mathrm{~mL}$ a diferentes concentraciones de bagazo (30, 40 y $50 \mathrm{mg} / \mathrm{mL})$.

$[$ Glucos $a]=\frac{A b s-0,004}{0,3084}$ 


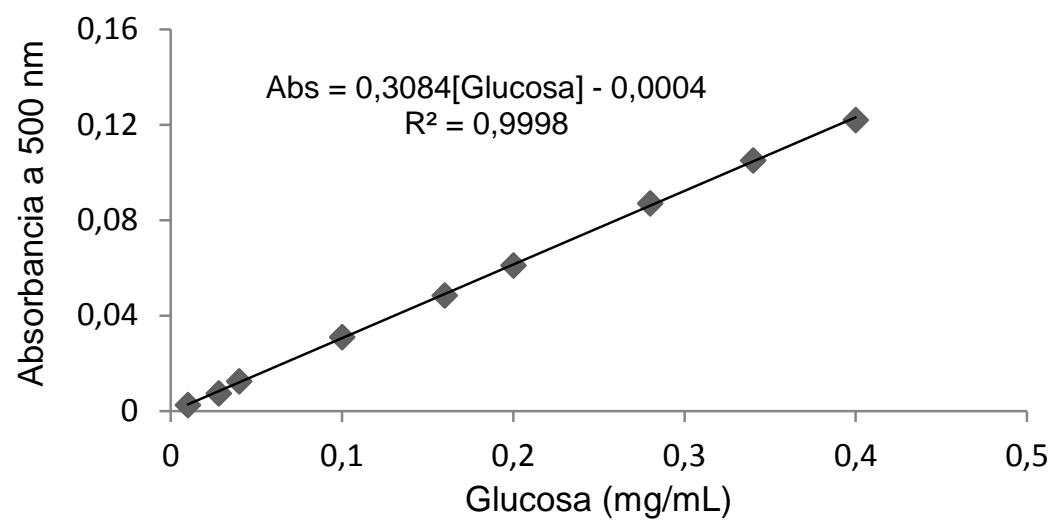

Fig. 1: Curva de calibrado del método analítico empleado para la determinación de glucosa.

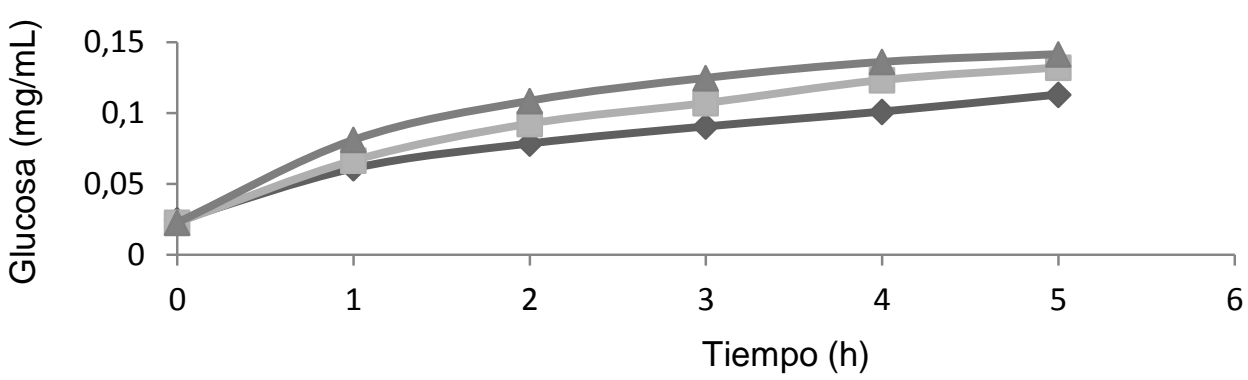

(a)

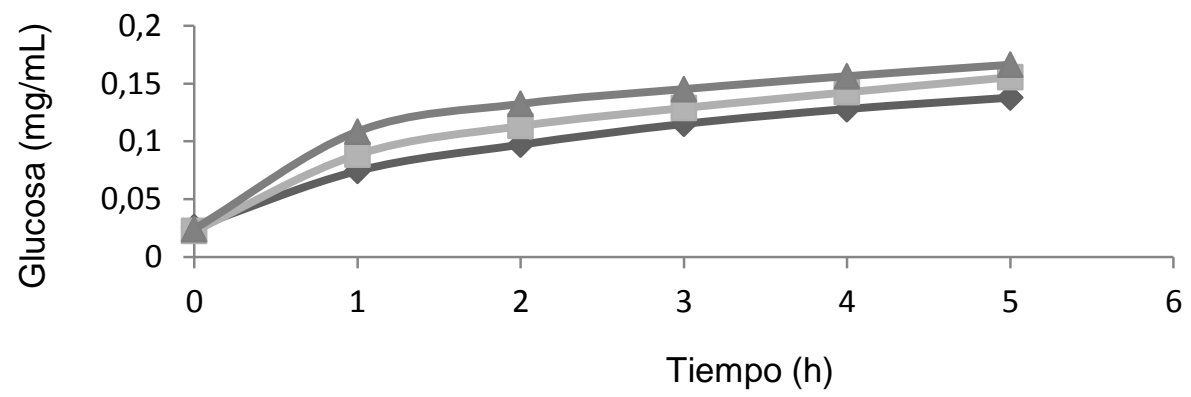

(b)

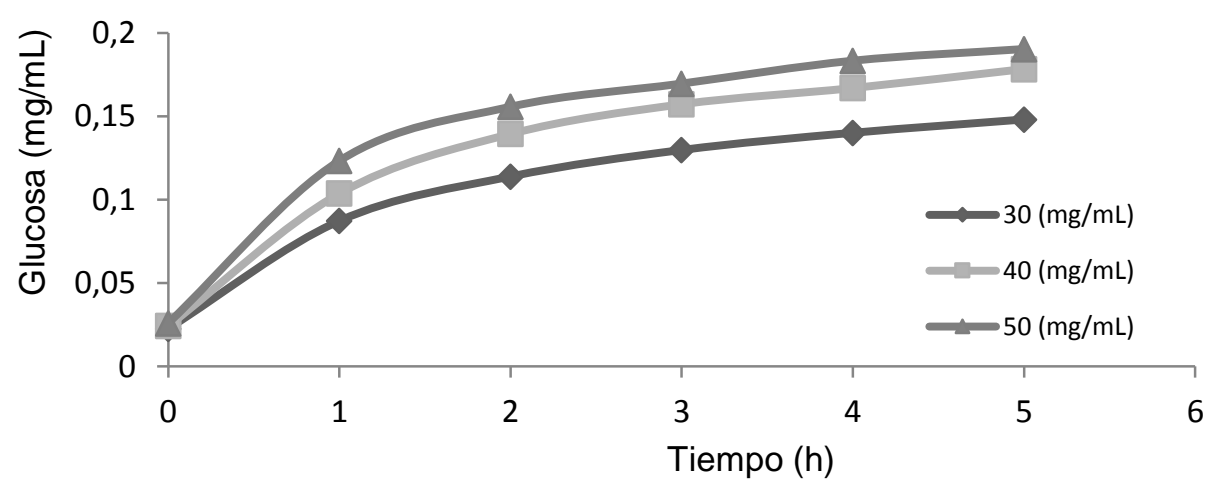

(c)

Fig. 2: Avance de reacción del bagazo de caña a una dosificación de enzima del (a) $0,210 \mathrm{ml}$, (b) $0,420 \mathrm{ml}$ y (c) $0,840 \mathrm{~mL}$. 
A través de cada uno de estos diagramas se puede ver claramente como la velocidad de formación del producto en la primera hora es mayor que en las horas siguientes. Esto se debe a que al adicionar la enzima al bagazo los centros activos de esta se encuentran desocupados, y en esos momentos entran en contacto con el bagazo todos los centros activos de la enzima, reaccionando con el sustrato que se encuentra en mayor proporción, provocando que se genere un rápido incremento en la velocidad de formación del producto (Goldbeter, 2013; Tzafriri y Edelman, 2007). Al transcurrir el tiempo la cantidad de sustrato que la enzima puede llegar a degradar va disminuyendo, generando a la vez una reducción en la velocidad de formación de producto. También se observó que al incrementarse la concentración de sustrato, manteniendo la dosificación de enzima constante, la concentración de glucosa o la velocidad de formación de producto aumentan, debido a que existe una mayor cantidad de sustrato que la enzima puede llegar a degradar. Se pude observar claramente que la mayor concentración de glucosa obtenida se encuentra a una concentración de sustrato inicial [So] $=50(\mathrm{mg} / \mathrm{mL})$ y una dosificación de enzima de $0,840 \mathrm{~mL}$ con un resultado de $0,1902 \mathrm{mg} / \mathrm{mL}$. También se puede analizar que al incrementar la dosificación de enzima manteniendo la [So] constante, la concentración de glucosa va aumentando.

Cálculo De la $V_{0}$ de la reacción enzimática y valores de $K_{m}$ y $V_{\max }$

Los datos de $V_{0}$ obtenidos a concentraciones de 30,40 y $50 \mathrm{mg} / \mathrm{mL}$ con dosificaciones de enzima constante de $0,210,0,420$ y $0,840 \mathrm{mg} / \mathrm{mL}$ se muestran en la Tabla 1 . A través de estos valores de $\mathrm{V}_{0}$ y [ $\mathrm{S}_{0}$ ] sé grafica la ecuación de Michaelis-Menten, para él cálculo de los valores de $K_{m}$ y $V_{\max }$ los cuales se observan en la Figura 3. En la Tabla 2 se presentan de forma resumida los datos de $K_{m}$ y $V_{\max }$ para las dosificaciones de enzima según la ecuación de Michaelis-Menten.

Tabla 1: Vo para una dosificación de enzima de 0,210 $\mathrm{ml}$ a diferentes concentraciones es de bagazo.

\begin{tabular}{|c|c|c|c|}
\hline & \multicolumn{2}{|c|}{ Vo $\left(\mathrm{mg} / \mathrm{mL}^{*} \mathrm{~s}\right)$} & $0,840 \mathrm{mg} / \mathrm{mL}$ \\
\hline Bagazo $(\mathrm{mg} / \mathrm{mL})$ & $0,210 \mathrm{mg} / \mathrm{mL}$ & $0,420 \mathrm{mg} / \mathrm{mL}$ & 0,06637 \\
\hline 30 & 0,04799 & 0,05967 & 0,07556 \\
\hline 40 & 0,05182 & 0,06618 & 0,08029 \\
\hline
\end{tabular}

En cada una de las representaciones graficas de la ecuación de Michaelis-Menten en donde se mantiene la dosificación de enzima constante variando la [So] de sustrato, se obtiene una curva hiperbólica, en donde al principio un aumento en la concentración de sustrato produce un incremento rápido en la velocidad de reacción, pero si se sigue aumentando la [So] la velocidad de reacción comienza a disminuir. La velocidad de reacción que se obtiene a altas [So] se define como la $V_{\max }$ de la reacción enzimática bajo condiciones especificas de $\mathrm{pH}$ y temperatura. El valor de la $\mathrm{V}_{\max }$ en cada una de las grafica nos indica que los centros activos de la enzima se encuentran saturados de sustrato a altas concentraciones de este (Bajzer y Strehler, 2012). En la Tabla 2 se observa como a una mayor dosificación de enzima la $V_{\max }$ es mayor. Esto se debe a que existe una mayor cantidad de sitos activos disponibles que deben saturarse de sustrato (Castro et al., 2013).

Con estas representaciones grafica de la ecuación de Michaelis-Menten se puede hallar los valores de $\mathrm{K}_{\mathrm{m}}$, que representa la concentración de sustrato a la que el $50 \%$ de los sitios activos de la enzima están ocupados con sustrato. En estas condiciones, la velocidad observada debe ser igual a la mitad de $\mathrm{V}_{\max }$. Al aumentar la concentración de enzima resulta un incremento en la $V_{\max }$ por lo tanto las tres curvas son semejantes en su forma, pero a mayor cantidad de enzimas tenemos curvas mas elevadas, esto se debe a que la velocidad solo depende de la concentración de sustrato. Los valores de V max nos indican la velocidad de catálisis cuando la enzima se encuentra saturada de sustrato (Goldbeter, 2013).

El valor de $\mathrm{K}_{\mathrm{m}}$ tiene un valor propio para cada enzima siendo así el parámetro que la caracteriza, por lo que este valor no tiene una variación significativa. $K_{m}$ mide la afinidad de la enzima con el sustrato, así para valore bajos, nos indica que la enzima se une con fuerza al sustrato, saturándose rápidamente el catalizador con pequeñas cantidades de sustrato. Para este análisis los valores de $\mathrm{K}_{\mathrm{m}}$ son muy altos lo que indica que la afinidad entre el bagazo y la enzima es baja por lo tanto las concentraciones de glucosa obtenida no son muy altas para este estudio (Goldbeter, 2013). 


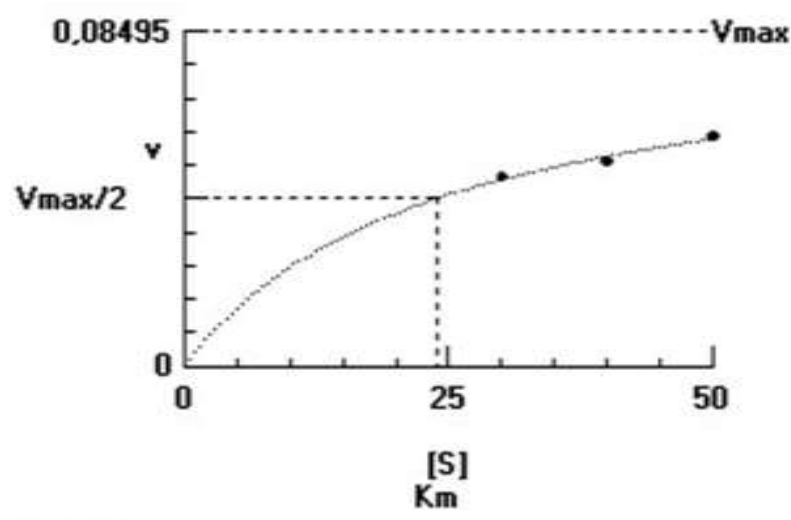

(a)

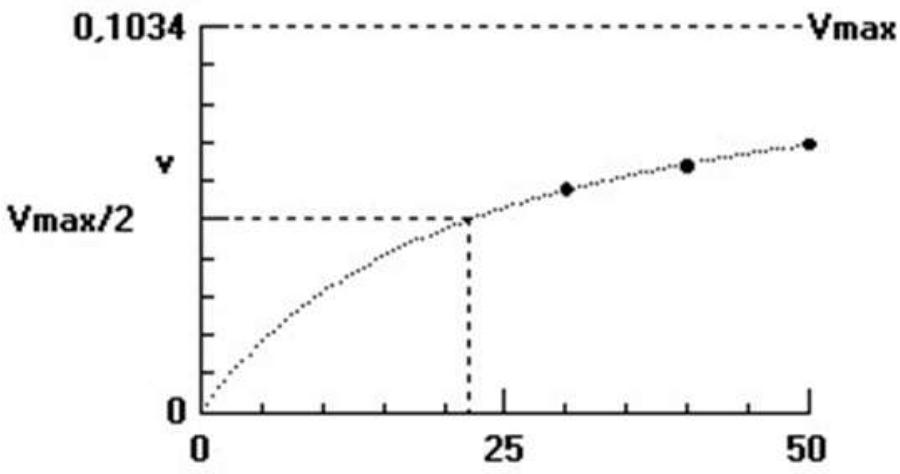

(b)

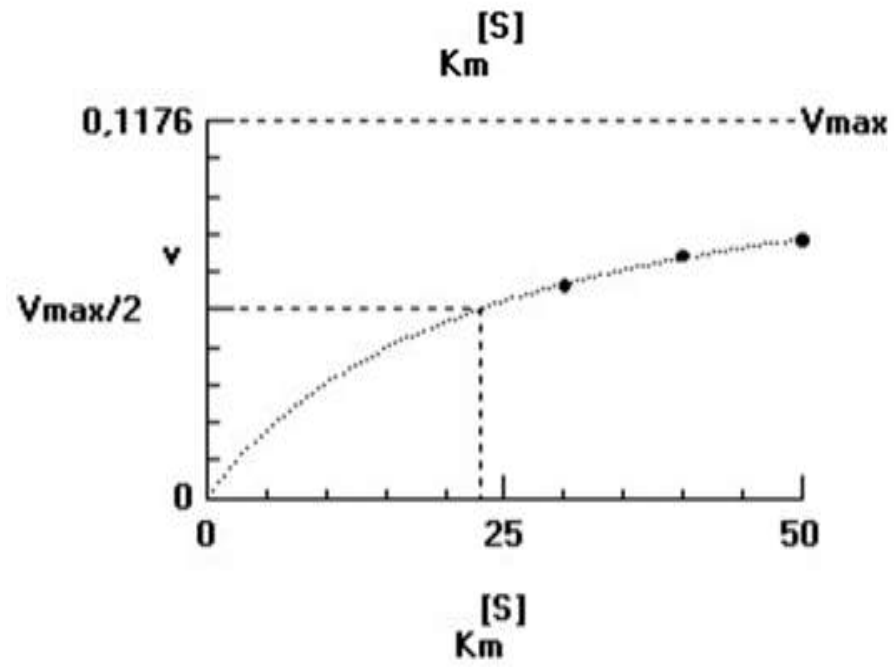

(c)

Fig. 3: Representación gráfica de la ecuación de Michaelis-Menten para el cálculo de $K_{m}$ y $V_{\max }$ a dosificaciones de enzima de (a) 0,210, (b) 0,420 y (c) 0,840 mg/mL.

Tabla 2: Datos de $V_{\max }$ y $\mathrm{K}_{\mathrm{m}}$ según Michaelis-Menten.

\begin{tabular}{|c|c|c|}
\hline Dosificación de enzima $(\mathrm{mL})$ & $\mathrm{V}_{\max }\left(\mathrm{mg} / \mathrm{mL}^{*} \mathrm{~s}\right)$ & $\mathrm{K}_{\mathrm{m}}(\mathrm{mg} / \mathrm{mL})$ \\
\hline 0,21 & 0,08495 & 22,86 \\
\hline 0,42 & 0,1034 & 22,7 \\
\hline 0,84 & 0,1176 & 22,86 \\
\hline
\end{tabular}




\section{CONCLUSIONES}

Al aumentar la concentración de sustrato [S $S_{0}$ manteniendo la dosificación de enzima constante, la velocidad de formación del producto [Glucosa] aumenta. Para este estudio de hidrólisis enzimática la dosificación de enzima mas adecuada fue la de $0,840 \mathrm{~mL}$. Según los datos obtenidos se observó que al aumentar la dosificación de enzima, la concentración final de glucosa después de 5 horas aumenta. La afinidad que existe entre la enzima y el sustrato es muy baja debido a los valores altos de $\mathrm{K}_{\mathrm{m}}$ que se encontraron.

\section{REFERENCIAS}

Agunsoye, J.O. y V.S. Aigbodion, Bagasse filled recycled polyethylene bio-composites: Morphological and mechanical properties study, Results in Physics: 3, 187-194 (2013).

Aigbodion, V.S. y otros tres autores, Potential utilization of solid waste (Bagasse Ash), Journal of Minerals \& Materials Characterization \& Engineering: 9, 67-77 (2010).

Bajzer, Z. y E.E. Strehler, About and beyond the Henri-Michaelis-Menten rate equation for single-substrate enzyme kinetics, Biochemical and Biophysical Research Communications: 417(3), 982-985 (2012).

Caspeta, L. y otros tres autores, Enzymatic hydrolysis at high-solids loadings for the conversion of agave bagasse to fuel ethanol, Applied Energy: 113, 277-286 (2014).

Castro, G.D., Dependencia de la dosis en los mecanismos de toxicidad y la evaluación de riesgo en toxicología. Acta bioquím. clín. Latinoam: 47(3), 0-0 (2013).

Cobas, M. y otros cuatro autores, Development of permeable reactive biobarrier for the removal of PAHs by Trichoderma longibrachiatum, Chemosphere: 91(5), 11-716 (2013).

Fangkum, A. y A. Reungsang, Biohydrogen production from sugarcane bagasse hydrolysate by elephant dung: effects of initial pH and substrate concentration, Int. J. Hydrogen Energy: 36, 8687-8696 (2011).

Freitas, M. y J.L. Colodette, Dissolving pulp production from sugar cane bagasse, Industrial Crops and Products: 52, 58-64 (2014).

Goldbeter, A., Oscillatory enzyme reactions and Michaelis-Menten kinetics, Febs Letters: 587(17), 27782784 (2013).

Guerrero, E.M., Cinética enzimática del bagazo de caña para la producción de glucosa utilizando la enzima Trichoderma longibrachiatum, Tesis de pregrado, Facultad de ingenieria Química, Universidad de San Buenaventura, Cartagena, Colombia (2004).

Lo, Y.C. y otros cinco autores, Biohydrogen production from cellulosic hydrolysate produced via temperature-shift-enhanced bacterial cellulose hydrolysis, Bioresour. Technol: 100, 5802-5807 (2009).

Lo, Y.C. y otros cuatro autores, Dark $\mathrm{H}_{2}$ fermentation from sucrose and xylose using $\mathrm{H}_{2}$-producing indigenous bacteria: feasibility and kinetic studies, Water Res: 42(4-5), 827-842 (2008).

Martínez, B., D. Infante, y Y. Reyes, Trichoderma spp. y su función en el control de plagas en los cultivos. Rev. Protección Veg: 28(1), pp. 1-11 (2013).

Panagiotopoulos, I.A. y otros seis autores, Biodiesel and biohydrogen production from cotton- seed cake in a biorefinery concept, Bioresour. Technol: 136, 78-86 (2013).

Pattra, S. y otros tres autores, Biohydrogen production from the fermentation of sugarcane bagasse hydrolysate by Clostridium butyricum, Int. J. Hydrogen Energy: 33, 6058-6065 (2008).

Peng, F. y otros cinco autores, Comparative study of hemicelluloses obtained by graded ethanol precipitation from sugarcane bagasse, J. Agric. Food Chem: 57, 6305-6317 (2009).

Rosales, E., M. Pazos y M. Á. Sanromán, Feasibility of Solid-State Fermentation Using Spent FungiSubstrate in the Biodegradation of PAHs, CLEAN - Soil, Air, Water: 41(6), 610-615 (2013). 
Sposina, R.T. y otros seis autores, Use of cellobiohydrolase-free cellulase blends for the hydrolysis of microcrystalline cellulose and sugarcane bagasse pretreated by either ball milling or ionic liquid [Emim][Ac], Bioresource Technology: 149, 551-555 (2013).

Stork, J. y otros cuatro autores, Regional examination shows potential for native feedstock options for cellulosic biofuel production, GCB Bioenergy: 1, 230-239 (2009).

Tzafriri, A.R. y E.R. Edelman, Quasi-steady-state kinetics at enzyme and substrate concentrations in excess of the Michaelis-Menten constant Journal of Theoretical Biology: 245(4), 737-748 (2007).

Wolf, L.D., Organosolv pre-treatment of sugar cane bagasse for ethanol production and xylooligomers obtainment, Master thesis, Federal University of São Carolos, São Carolos, Brasil, 147 pp (2011). 